\title{
Elimination Pathways of Nanoparticles
}

Wilson Poon ${ }^{1,2}$, Yi-Nan Zhang*1,2, Ben Ouyang*1,2,3, Benjamin R. Kingston ${ }^{1,2}$, Jamie L. Y. Wu ${ }^{1,2}$, Stefan Wilhelm ${ }^{4,5}$, Warren C. W. Chan ${ }^{1,2,6,7,8}$

${ }^{1}$ Institute of Biomaterials \& Biomedical Engineering, University of Toronto, Toronto, ON M5S 3G9, Canada

${ }^{2}$ Terrence Donnelly Centre for Cellular \& Biomolecular Research, University of Toronto, Toronto, ON M5S 3E1, Canada

${ }^{3} \mathrm{MD} / \mathrm{PhD}$ Program, University of Toronto, Toronto, ON M5S 1A8, Canada

${ }^{4}$ Stephenson School of Biomedical Engineering, University of Oklahoma, Norman, OK 73019, USA

${ }^{5}$ Stephenson Cancer Center, Oklahoma City, OK 73104, USA

${ }^{6}$ Department of Chemical Engineering \& Applied Chemistry, University of Toronto, Toronto, ON M5S 3E5, Canada

${ }^{7}$ Department of Materials Science \& Engineering, University of Toronto, Toronto, ON M5S 1A1, Canada

${ }^{8}$ Department of Chemistry, University of Toronto, Toronto, ON, M5S 3H6, Canada

*These authors contributed equally to this work 


\section{Table of Contents}

1. AuNP physico-chemical properties Table T1

2. AuNP synthesis Fig. S1

3. Functionalization of AuNPs Fig. S2

4. Ex vivo fluorescence imaging of BALB/c mice livers post-injection of AF750-PEG-AuNPs Fig. S3

5. TEM micrographs of AuNPs remaining intact along the hepatobiliary transit pathway in BALB/C mice.

6. Quantification of AuNP size in bile and feces post-injection into BALB/c mice Fig. S5

7. Weight of feces collected daily over experimental period Fig. S6

8. Organ-level redistribution of $50 \mathrm{~nm}$ AuNPs in BALB/c mice Fig. 57

9. Clodronate-liposome treatment does not affect hepatic $B$ cells and stellate cells

10. Segmentation of liver sinusoids from CD209b immunohistology slides by llastik Fig. 58

11. Subcellular visualization of $50 \mathrm{~nm}$ AuNPs sequestered by Kupffer cells Fig. 59

12. Dose optimization of injected AuNPs in BALB/c mice for reliable detection by ICP-MS Fig. S10

13. Hepatobiliary elimination with changed order of AuNP \& clodronate-liposome injection .. Fig. S12

14. Blood concentration of AuNPs following release from liver using clodronate-liposomes.... Fig. S13

15. Characterization of liver sinusoidal endothelial cell fenestrae of control PBS-liposome and clodronate-liposome treated BALB/c mice

16. Quantification of how depletion of Kupffer cells improves fecal elimination of AuNPs Fig. S15

17. Organ-level distribution of different AuNP sizes for control \& pre-depletion groups Fig. S16

18. Cumulative AuNP ID\% eliminated in 14 days in control BALB/c mice is a function of AuNP size

19. Subcellular visualization of $15 \mathrm{~nm}$ AuNPs undergoing hepatobiliary processing by TEM ..... Fig. S18

20. Subcellular visualization of $100 \mathrm{~nm}$ AuNPs undergoing hepatobiliary processing by TEM ... Fig. S19

21. Subcellular visualization of $200 \mathrm{~nm}$ AuNPs in liver sinusoid by TEM... Fig. S20

22. Characterization \& synthesis of Cy3/Cy5 dual-labelled degradable liposomes Fig. S20 
Table T1. AuNP physico-chemical properties

\begin{tabular}{|c|c|c|c|c|c|}
\hline \multicolumn{2}{|c|}{ Nanoparticle } & \multicolumn{4}{|c|}{ Physico-chemical properties } \\
\hline Size & Surface ligands & $\begin{array}{l}\text { Inorganic } \\
\text { diameter }^{\mathrm{a}}(\mathrm{nm})\end{array}$ & $\begin{array}{l}\text { Hydrodynamic } \\
\text { diameter }(\mathrm{nm})\end{array}$ & $\begin{array}{l}\text { Zeta potential } \\
(\mathrm{mV})\end{array}$ & $\lambda_{\text {LSPR }}(\mathrm{nm})$ \\
\hline 4 & $\begin{array}{l}\text { BSPP } \\
\text { PEG }\end{array}$ & $4.4 \pm 0.6$ & $\begin{array}{l}4.8 \pm 0.8 \\
11.5 \pm 0.5\end{array}$ & $\begin{array}{c}-26.5 \pm 1.6 \\
-5.0 \pm 1.1\end{array}$ & 512 \\
\hline 15 & $\begin{array}{l}\text { Citrate } \\
\text { AF750 - PEG }\end{array}$ & $15.5 \pm 1.4$ & $\begin{array}{l}21.1 \pm 0.3 \\
51.8 \pm 0.3\end{array}$ & $\begin{array}{l}-12.7 \pm 1.1 \\
-1.5 \pm 0.8\end{array}$ & 519 \\
\hline 50 & $\begin{array}{l}\text { Citrate } \\
\text { AF750 - PEG }\end{array}$ & $48.4 \pm 6.1$ & $\begin{array}{l}53.6 \pm 0.3 \\
89.0 \pm 1.0\end{array}$ & $\begin{array}{l}-17.7 \pm 0.1 \\
-2.6 \pm 1.7\end{array}$ & 535 \\
\hline 100 & $\begin{array}{l}\text { Citrate } \\
\text { AF750 - PEG }\end{array}$ & $109.8 \pm 11.7$ & $\begin{array}{l}121.4 \pm 1.7 \\
167.9 \pm 1.4\end{array}$ & $\begin{array}{l}-32.7 \pm 1.7 \\
-1.5 \pm 0.5\end{array}$ & 576 \\
\hline 200 & $\begin{array}{l}\text { Citrate } \\
\text { AF750 - PEG }\end{array}$ & $220.9 \pm 10.0$ & $\begin{array}{l}212.6 \pm 1.0 \\
293.1 \pm 9.6\end{array}$ & $\begin{array}{l}-25.7 \pm 1.1 \\
-2.6 \pm 5.8\end{array}$ & 570 \\
\hline
\end{tabular}

a Inorganic diameter was measured using transmission electron microscopy and histogram distributions were compiled from the counting of ca. 100 particles using Image J software. For citrate stabilized AuNPs, measurements were acquired in $0.02 \%$ w/ $\mathrm{v}$ citrate solutions with the exception of $4 \mathrm{~nm}$ and $15 \mathrm{~nm}$ AuNPs; particles were measured as synthesized. For remaining AuNPs, hydrodynamic diameters were measured in $0.02 \% \mathrm{w} / \mathrm{v}$ citrate solution and zeta potentials were recorded in HEPES buffer. Precision reported is the standard deviation of triplicate measurements, nanoparticle samples having a PDI >0.1 were discarded.

Abbreviations: $B S P P=$ bis(p-sulfonatophenyl)phenylphosphine dihydrate dipotassium salt; $P E G=$ polyethylene glycol; $A F 750=$ Alexafluor 750 fluorophore 
A

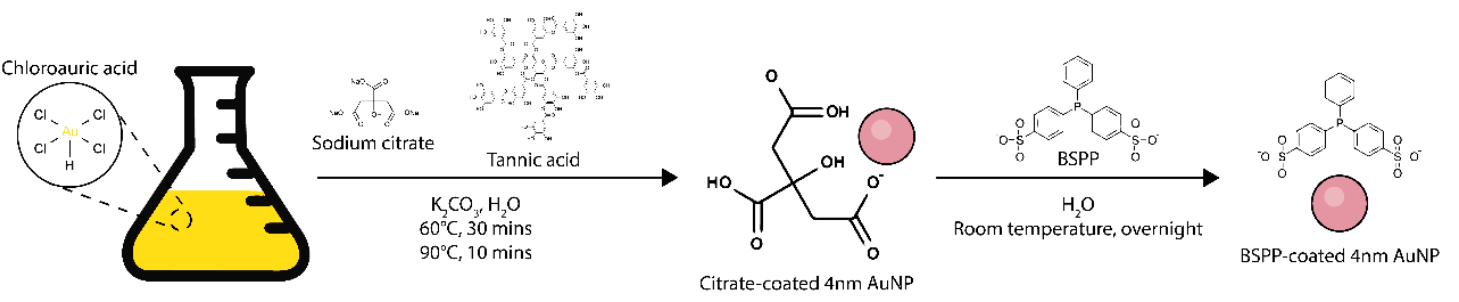

B
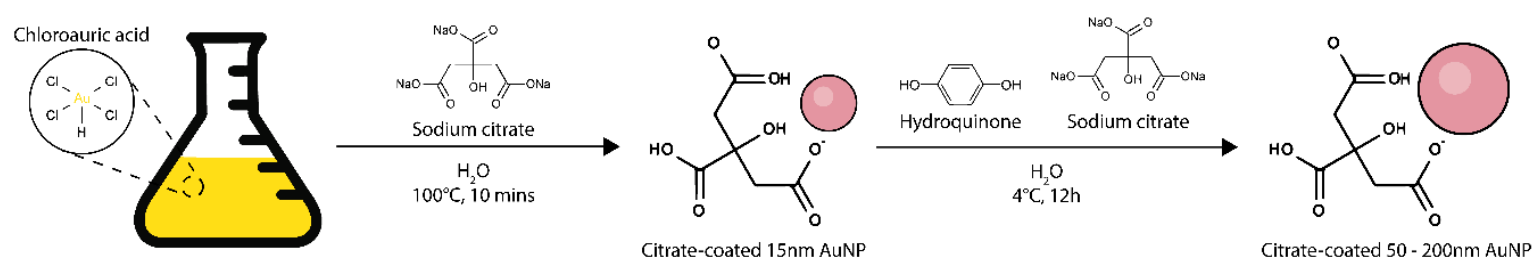

Fig. S1 - AuNP synthesis

(A) Schematic for synthesis of $4 \mathrm{~nm}$ AuNP by reduction of chloroauric acid by sodium citrate, tannic acid, and potassium carbonate; followed by surface modification by BSPP (bis(p-sulfonatophenyl)phenylphosphine dihydrate dipotassium salt); (B) Schematic for synthesis of AuNPs larger than $4 \mathrm{~nm}$ by Frens' method, followed by seed-mediated growth using Perrault's method.

A

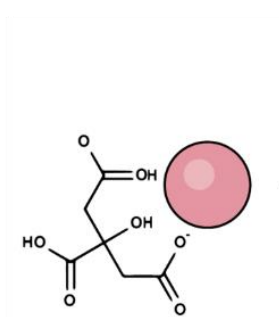

Citrate-AuNP

B

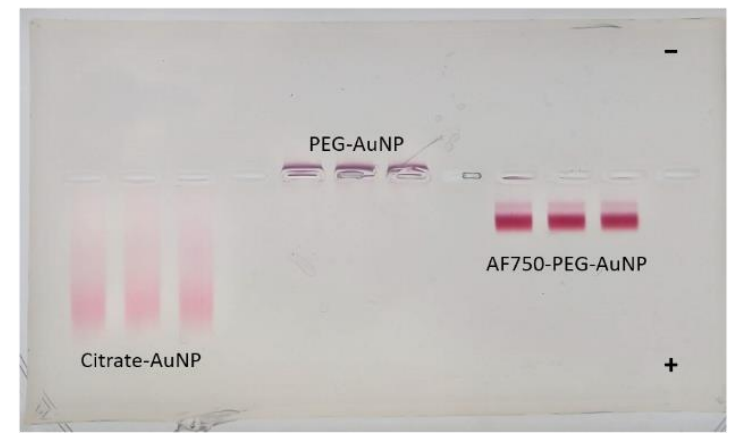

20\% 10kDa amino PEG

PEG-AuNP

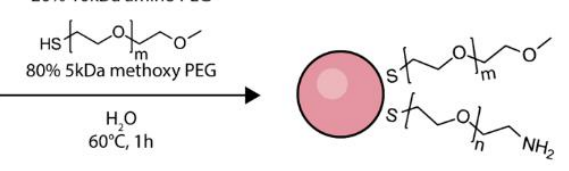

C

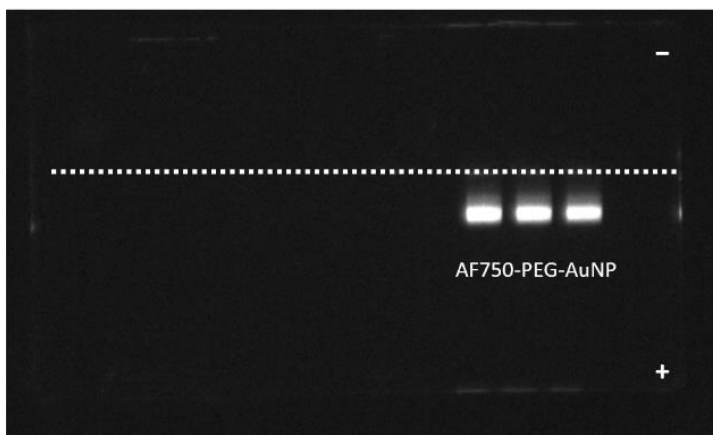

Fig. S2 - Functionalization of AuNPs

(A) Schematic of surface modification of AuNPs with PEG and AF750 fluorophore; (B) agarose gel electrophoretogram of $50 \mathrm{~nm}$ citrate AuNPs, PEG-AuNPs, and AF750-PEG-AuNPs; (C) Corresponding fluorescence agarose gel electrophoretogram (730 nm excitation, $790 \mathrm{~nm}$ emission) of $50 \mathrm{~nm}$ citrate AuNPs, PEG-AuNPs, and AF750-PEG-AuNPs. 


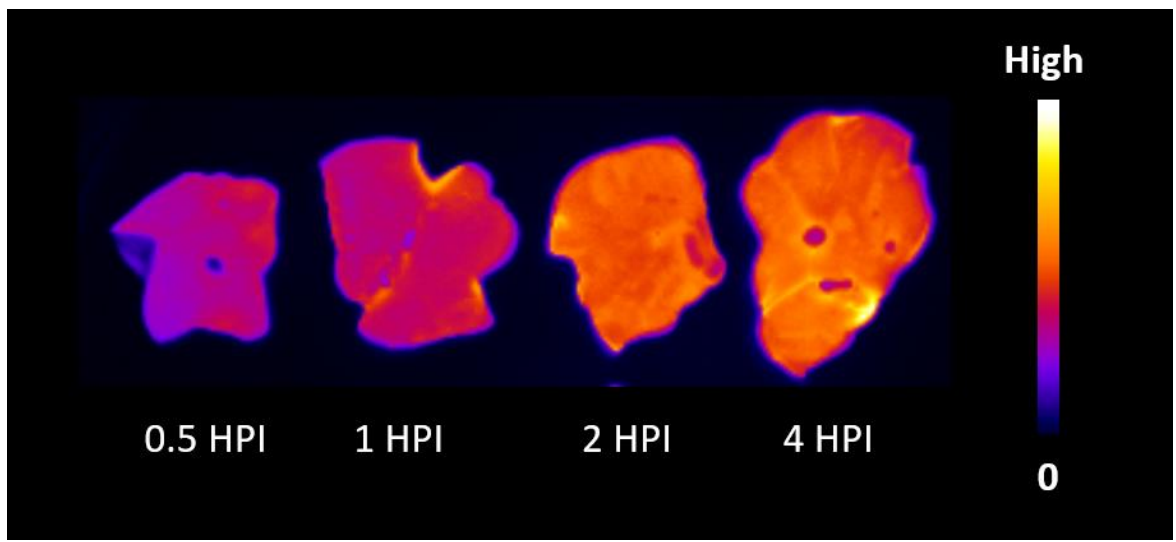

Fig. S3 - Ex vivo fluorescence imaging of BALB/c mice livers post-injection of AF750-PEG-AuNPs Ex-vivo fluorescence imaging of BALB/c mice livers (left to right) 0.5 hours, 1 hour, 2 hours, and 4 hours post-injection (HPI) of AF750-PEG-AUNPs. 


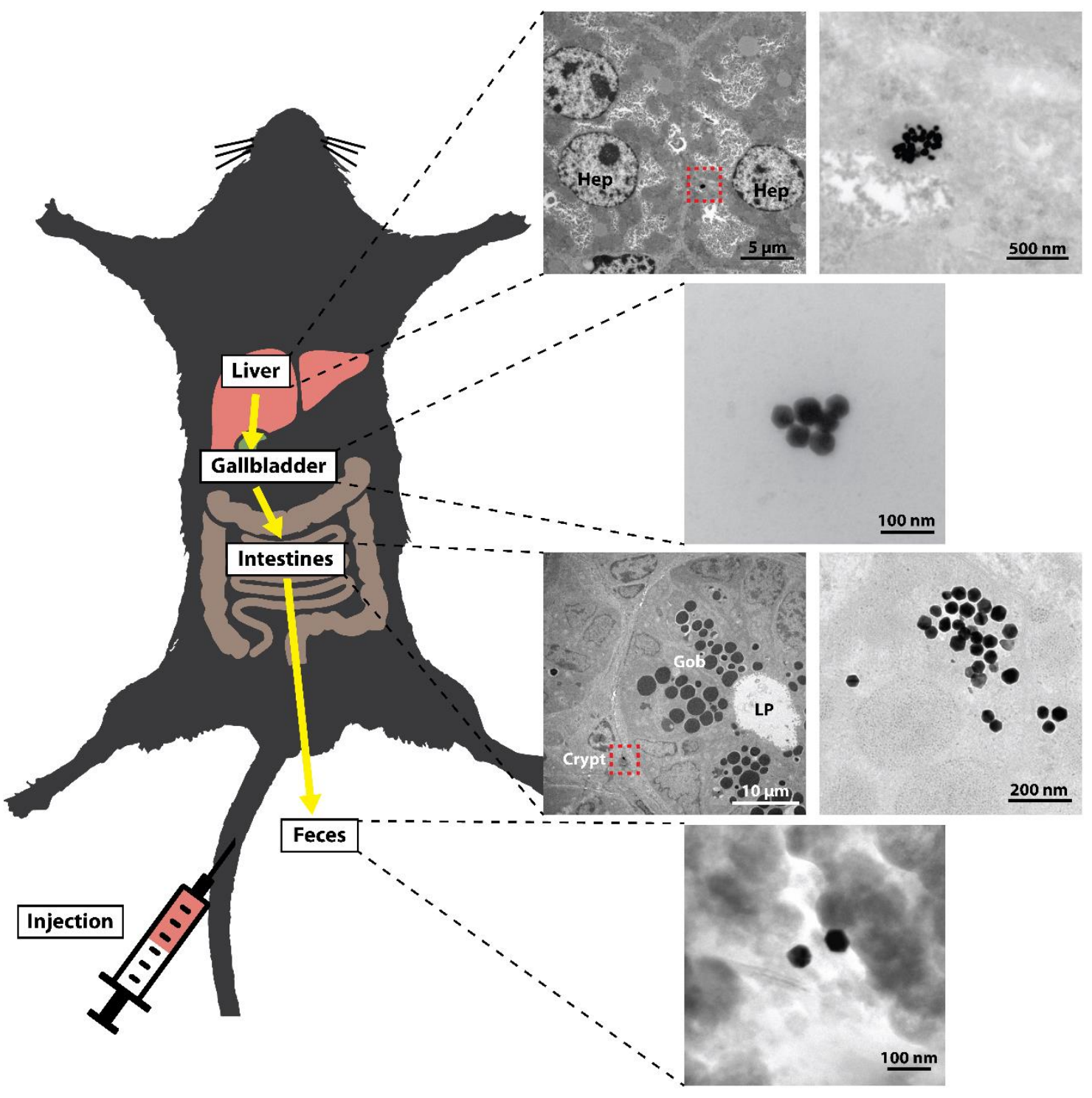

Fig. S4 - TEM micrographs of AuNPs remaining intact along the hepatobiliary transit pathway in BALB/c mice Representative TEM images of $50 \mathrm{~nm}$ AuNPs remaining intact along the hepatobiliary transit pathway in BALB/c mice 14 days post-injection. Right panels for the liver and intestines are magnified regions of the corresponding areas indicated by the red dotted squares on their respective left panels. Hep = hepatocyte, Gob = goblet cell, $L P=$ lamina propria, Crypt = intestinal crypt. 


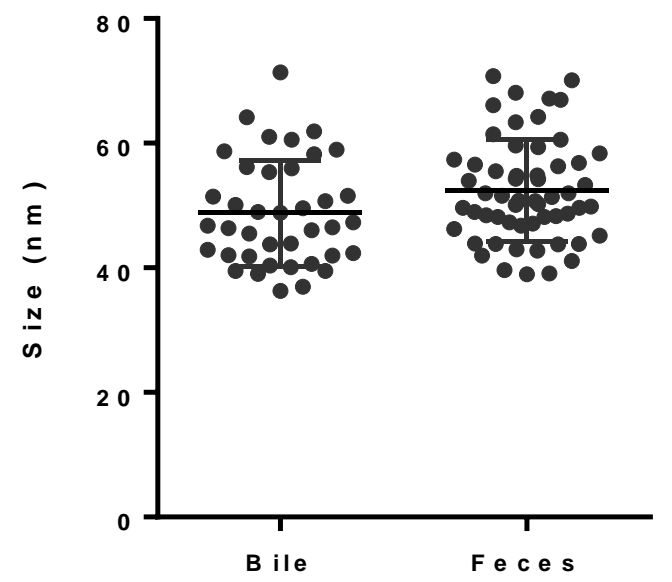

Fig. S5 - Quantification of AuNP size in bile and feces post-injection into BALB/c mice Quantification of $50 \mathrm{~nm}$ AUNP size in bile and feces 14 days post-injection into BALB/C mice as determined by TEM. Data is displayed as mean \pm standard deviation with a sample size of $n>39-57$.

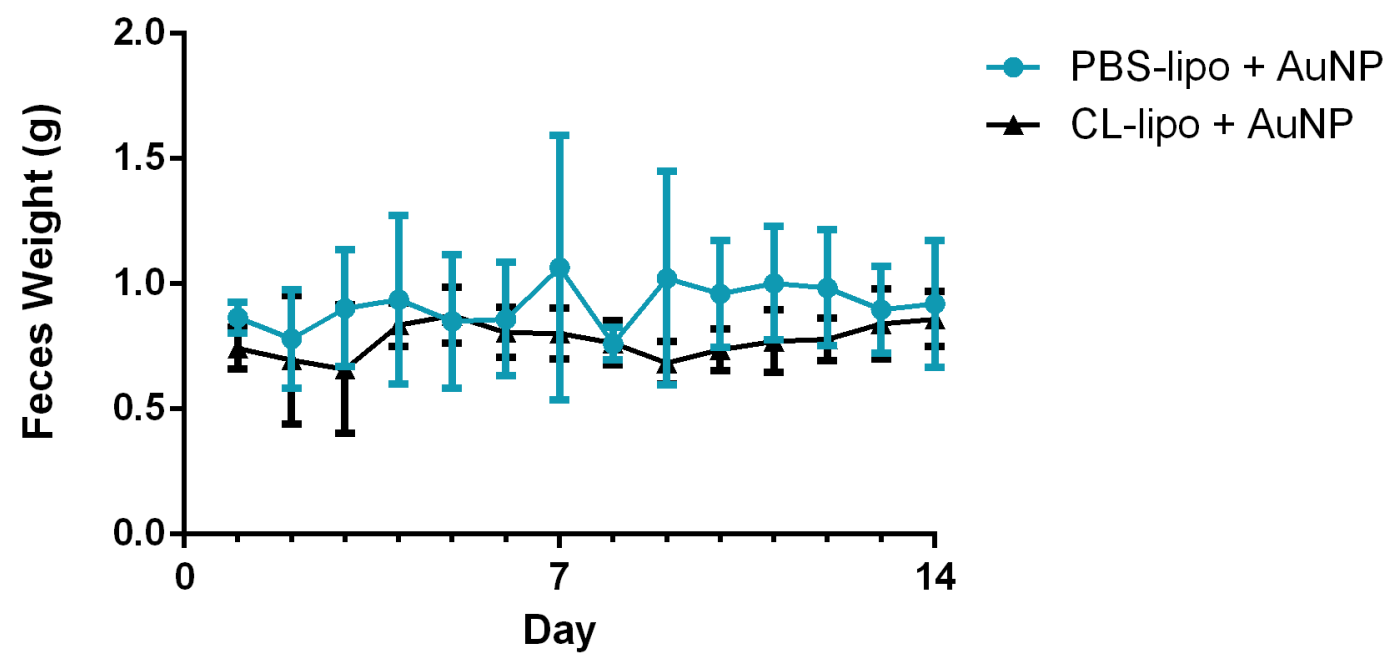

Fig. S6 - Weight of feces collected daily over experimental period Weight of feces collected daily for 14 days post-injection of $50 \mathrm{~nm}$ AuNPs from PBS-liposomes (PBS-lipo) + AuNPs and CLliposomes (CL-lipo) + AuNPs BALB/c mice. Data is displayed as mean \pm standard deviation with a sample size of $n=3-4$. 


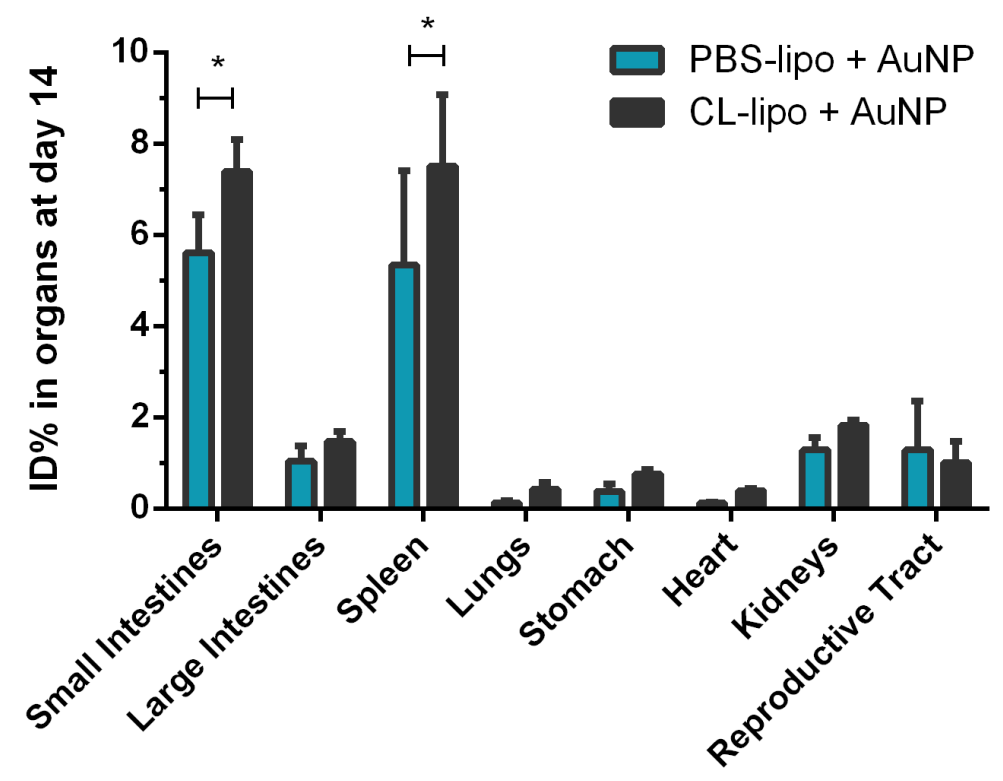

Fig. S7 - Organ-level redistribution of $\mathbf{5 0} \mathbf{~ n m}$ AuNPs in BALB/c mice

ID\% of AUNPs in each major organ of BALB/C mice 14 days post-injection of $50 \mathrm{~nm}$ AuNPs for control PBS-liposome (PBS-lipo) and clodronate-liposome (CL-lipo) pre-depletion groups; Data is displayed as mean \pm standard deviation with a sample size of $n$ $=3-4$. Statistics determined by multiple t-test with Sidak-Bonferroni correction, ${ }^{*}=p<0.05$.
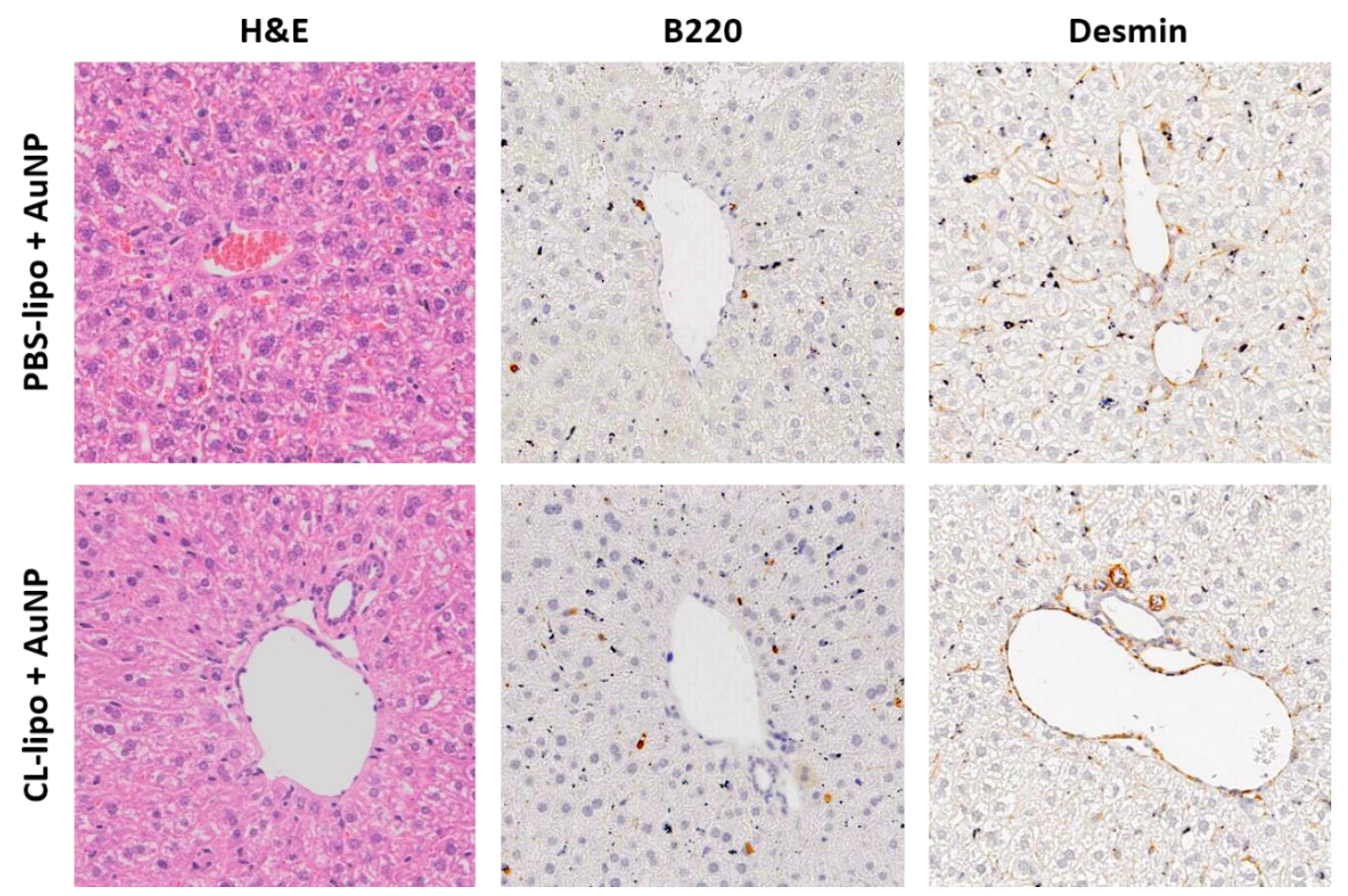

Fig. S8 - Clodronate-liposome treatment does not affect hepatic B cells and stellate cells Immunohistological sections at 10x magnification of clodronate-liposome (CL-lipo) pre-treated and control PBS-liposome (PBSlipo) pre-treated $B A L B / C$ mice livers 72 hours post-injection of liposomes; stained with hematoxylin \& eosin, B220 antibody (for liver $B$ cells), and desmin antibody (for hepatic stellate cells). 


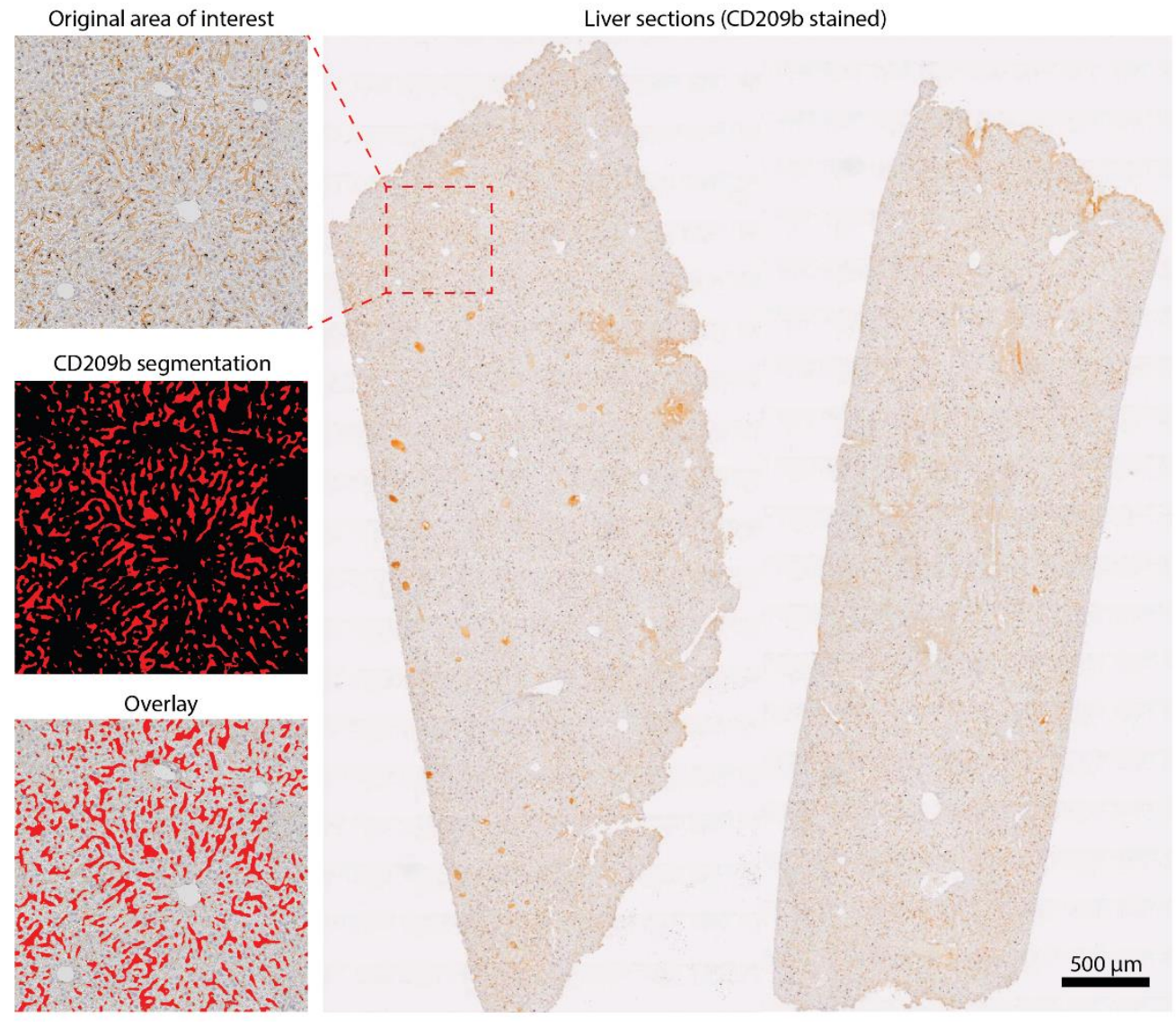

Fig. S9 - Segmentation of liver sinusoids from CD209b immunohistology slides by llastik CD209b immunohistology slides and segmentation of liver sinusoids by llastik of BALB/c livers. Original area of interest is shown with the red dotted square in CD209b stained liver sections. CD209b segmentation was performed by ilastik and FIJI was used to infill the sinusoidal volume. This results in the red area that we define as the intravascular region of the liver (includes the liver sinusoidal endothelium itself), and the black area that we define as the extravascular region of the liver (primarily the hepatocyte region only) as shown in the overlay. 

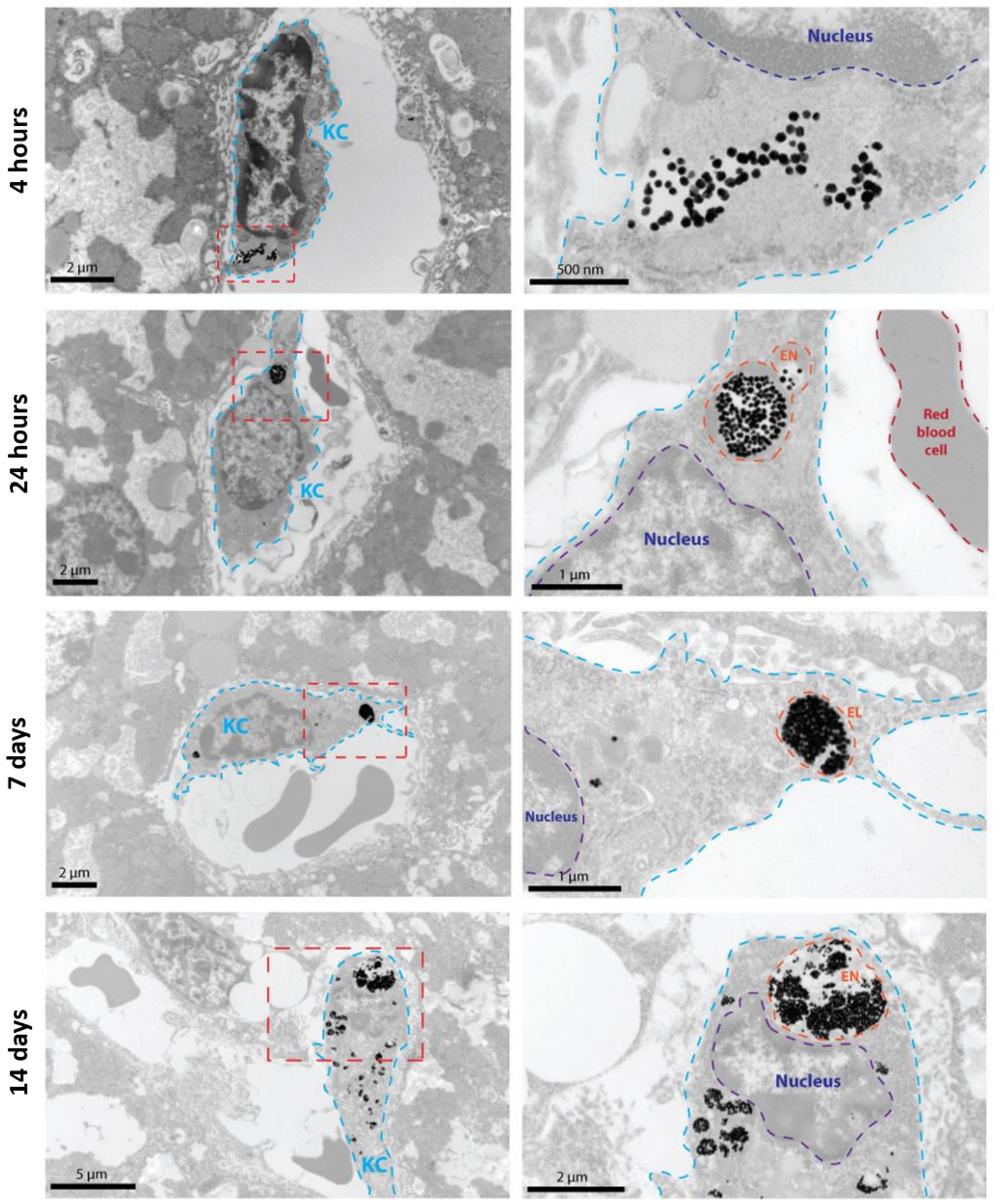

Fig. S10 - Subcellular visualization of $\mathbf{5 0} \mathbf{~ n m ~ A u N P s ~ s e q u e s t e r e d ~ b y ~ K u p f f e r ~ c e l l s ~}$

(Top to bottom) Representative TEM images of livers from control BALB/C mice at 4 hours, 24 hours, 7 days, and 14 days postinjection of $50 \mathrm{~nm}$ AuNPs. Right panels are magnified regions of the corresponding areas indicated by the red dotted squares. $K C$ $=$ Kupffer cell, $E N=$ endosome. 

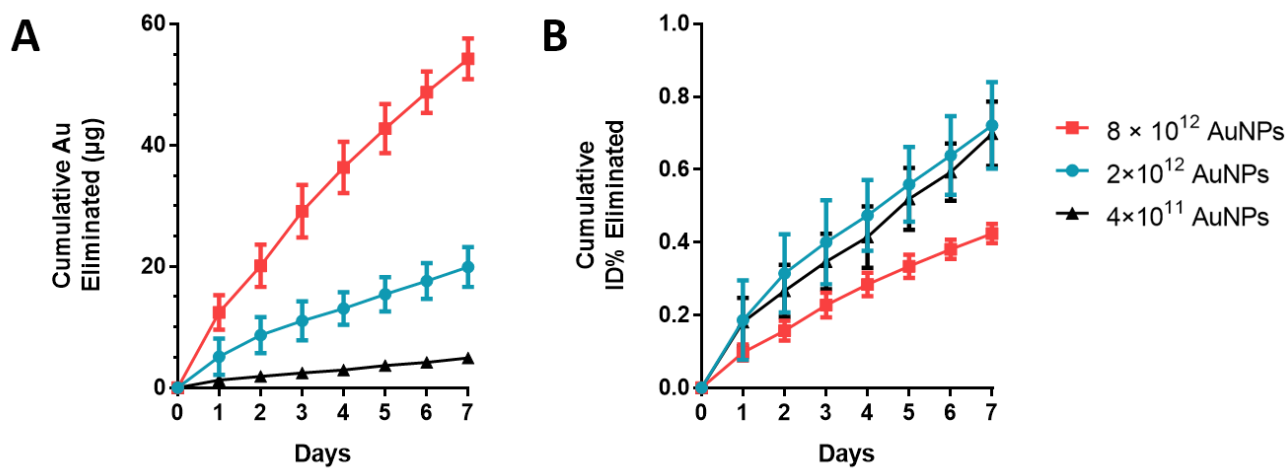

Fig. S11 - Dose optimization of injected AuNPs in BALB/c mice for reliable detection by ICP-MS

(A) Cumulative mass of gold eliminated from $B A L B / c$ mice over 7 days post-injection of $50 \mathrm{~nm}$ AuNPs at three different administered doses. (B) Cumulative ID\% eliminated from BALB/c animals over 7 days post-injection of $50 \mathrm{~nm}$ AuNPs at three different administered doses. BALB/c mice were not pre-treated with PBS-liposomes or clodronate-liposomes. 


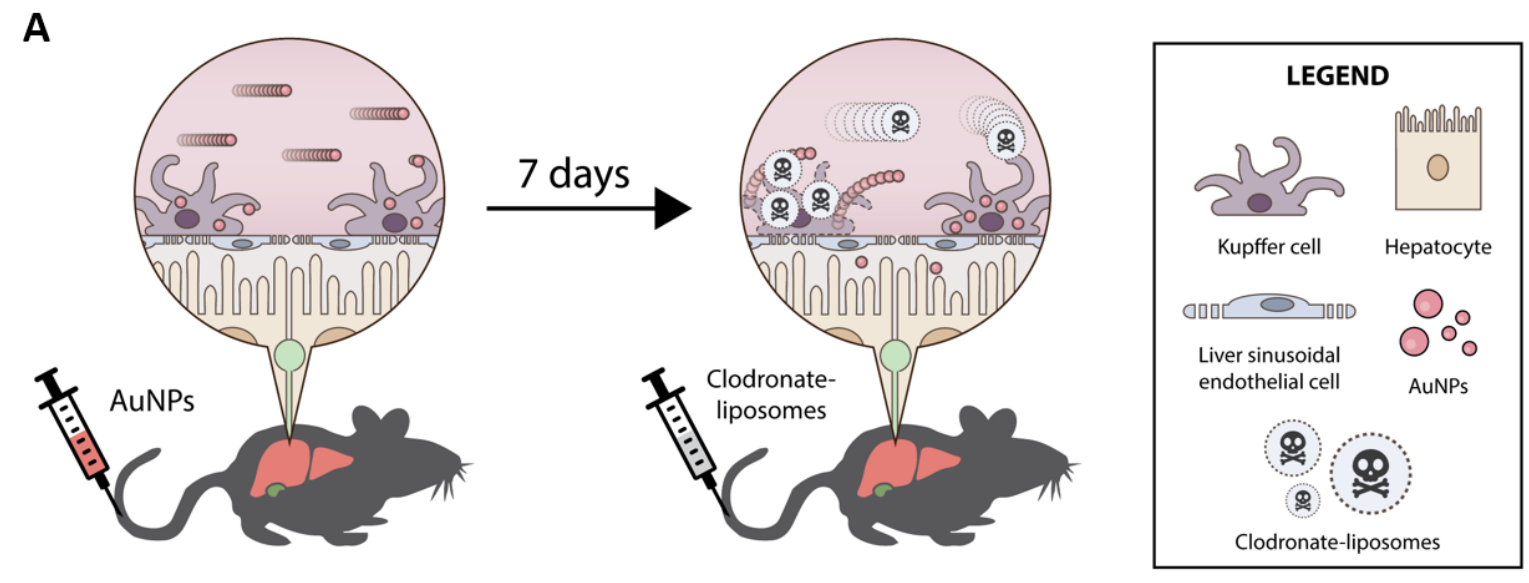

B

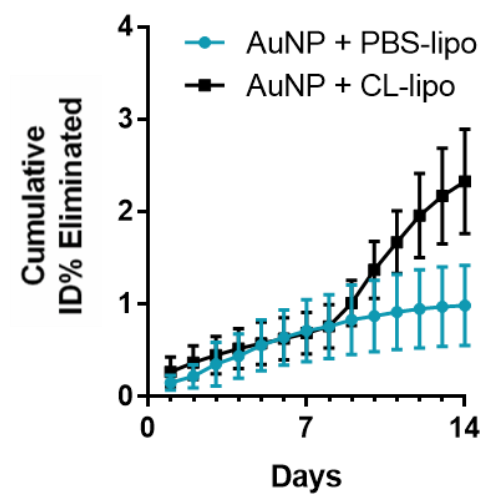

C

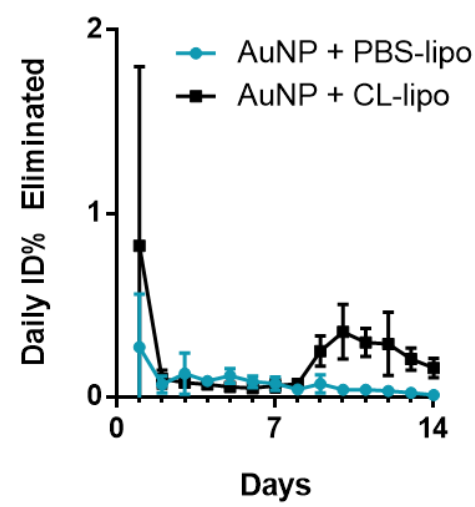

D

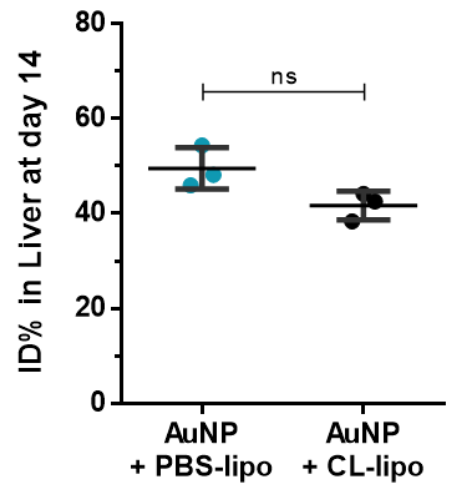

Fig. S12 - Hepatobiliary elimination with changed order of AuNP \& clodronate-liposome injection

(A) Schematic of the jailbreak experiment design to investigate Kupffer cells and nanoparticle hepatobiliary elimination. BALB/C mice are injected with $50 \mathrm{~nm}$ AuNPs first, AuNPs accumulate in Kupffer cells and clodronate-liposomes (CL-lipo) are injected on day 7 to cause Kupffer cell apoptosis to release sequestered AuNPs back into blood circulation; (B) Cumulative ID\% eliminated from BALB/c mice over 14 days post-injection of $50 \mathrm{~nm}$ AuNPs with PBS-liposomes (PBS-lipo) or clodronate-liposomes administered on day 7; (C) Daily rate of ID\% eliminated from BALB/c mice over 14 post-injection of $50 \mathrm{~nm}$ AuNPS with PBSliposomes or clodronate-liposomes administered on day 7; (D) ID\% of liver at the end of the experiment from BALB/C mice(14 days post-injection of $50 \mathrm{~nm}$ AuNPs). Data is displayed as mean \pm standard deviation with a sample size of $n=3$. Statistics determined by unpaired $t$-test with $n s=$ not statistically significant. 


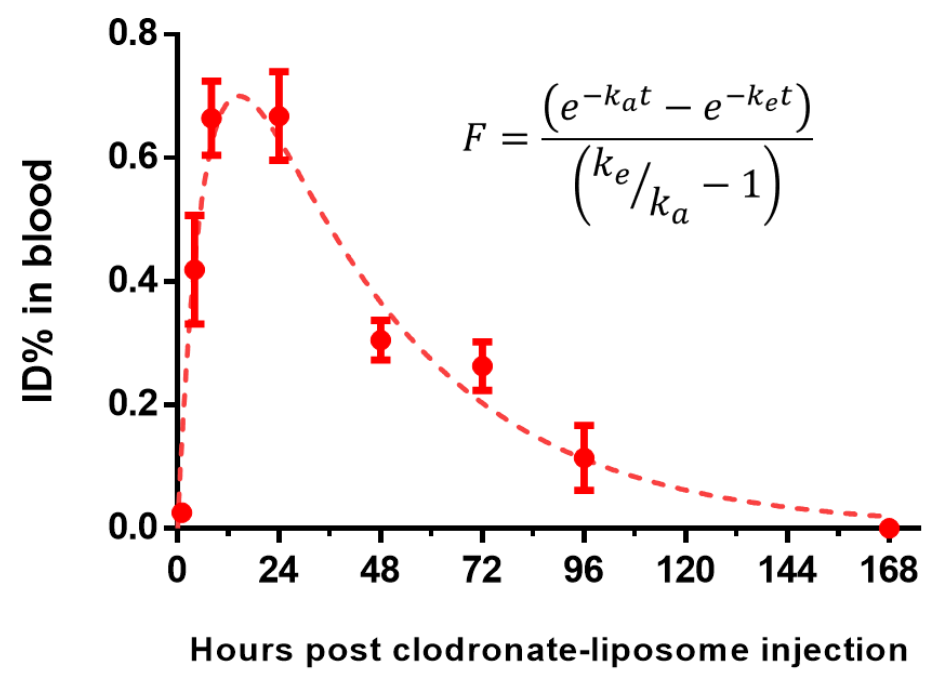

Fig. S13 - Blood concentration of AuNPs following release from liver using clodronate-liposomes

$B A L B / c$ mice are injected with $50 \mathrm{~nm}$ AuNPs on day 1, AuNPs accumulate in Kupffer cells and clodronate-liposomes (CL-lipo) are injected on day 7 to cause Kupffer cell apoptosis to release sequestered AuNPs back into blood circulation (same as shown in Fig. S12A). ID\% of $50 \mathrm{~nm}$ AuNPs in blood following release from liver Kupffer cells post-injection of clodronate-liposomes in BALB/C mice. Data is displayed as mean \pm standard deviation with a sample size of $n=4-6$. Data was fit to the shown conventional drug release profile equation with first-order elimination and first-order absorption. $F$ is the ID\% of AuNPs in blood, $t$ is time, $k_{e}$ is the elimination constant, and $k_{a}$ is the absorption constant. $C_{\max }$ is approximately $0.7 \%$ ID at 18 hours post-injection of clodronateliposomes, the half-life of AuNP liberation from Kupffer cells is approximately 4.6 hours, and the blood half-life of freed AuNPs is approximately 28.1 hours.
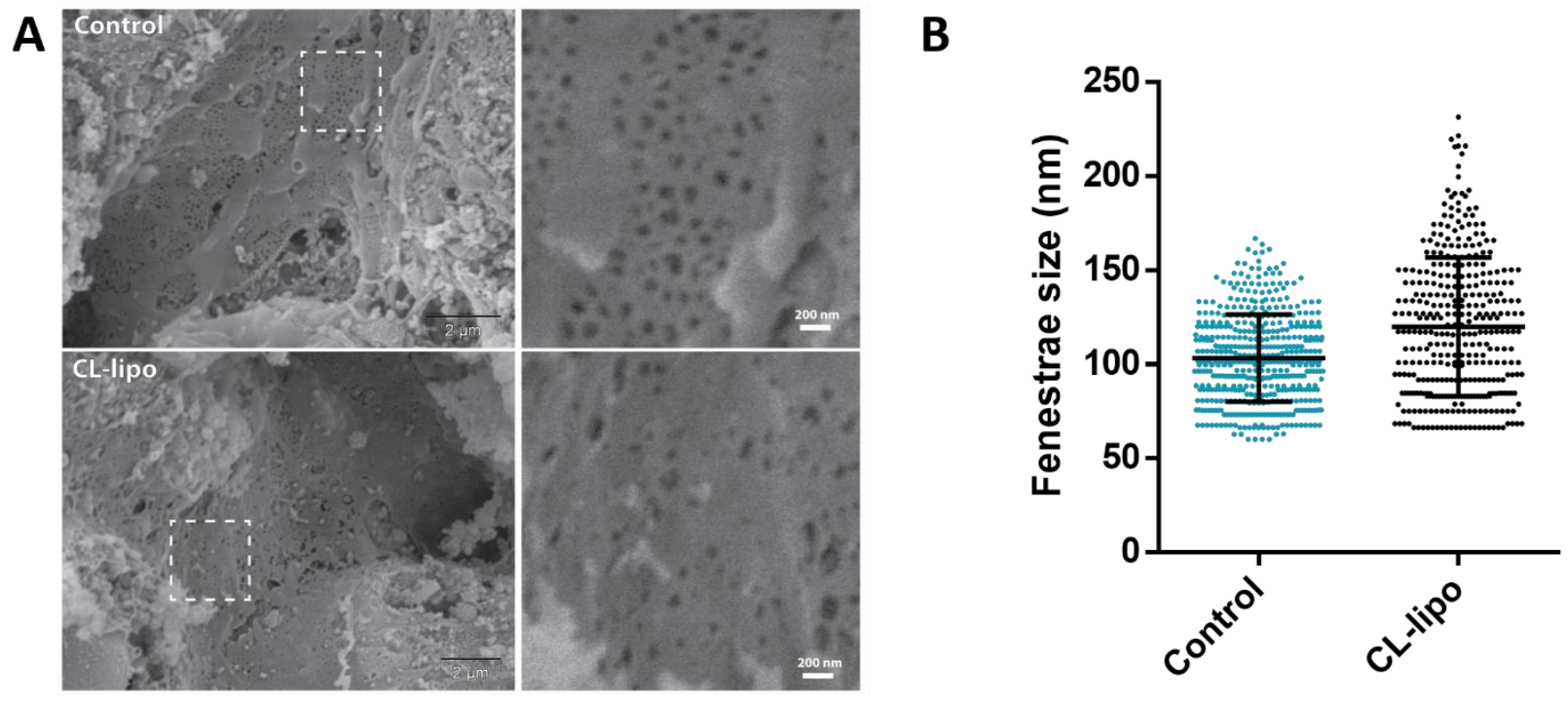

Fig. S14 - Characterization of liver sinusoidal endothelial cell fenestrae of control PBS-liposome and clodronate-liposome treated BALB/c mice

(A) Representative SEM images of liver sinusoids with no treatment (control) (top) and 48 hours post-injection of clodronateliposomes (CL-lipo) (bottom), right panel shows zoomed in section of left panel as indicated by the dotted white box; (B) Fenestrae size of liver sinusoidal endothelial cells in BALB/c mice before and after Kupffer cell depletion by clodronate-liposome treatment as determined by segmentation by llastik \& image analysis by FIJI (data is displayed as mean \pm standard deviation with \# of fenestrae observed = 353-483). 
A

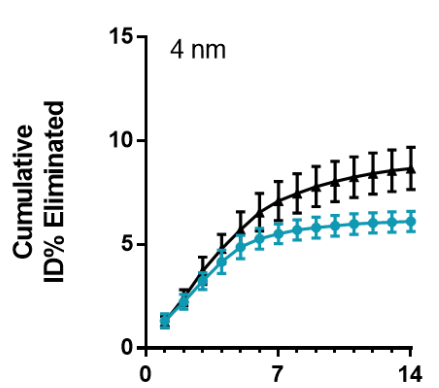

D

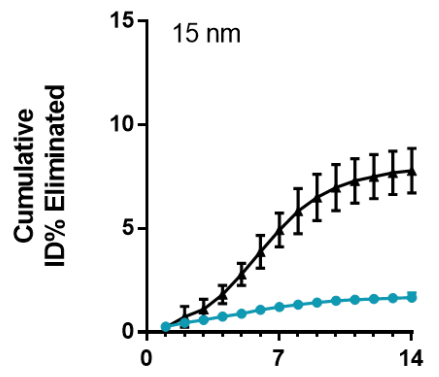

G

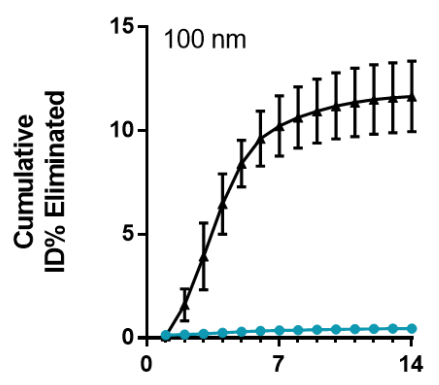

J

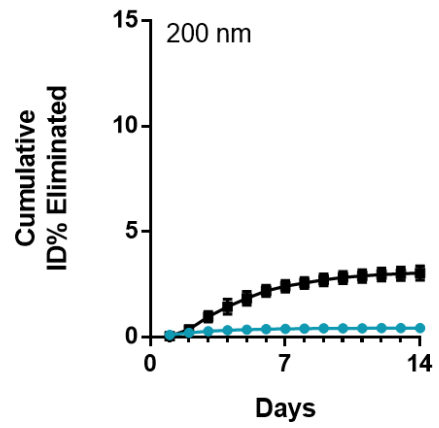

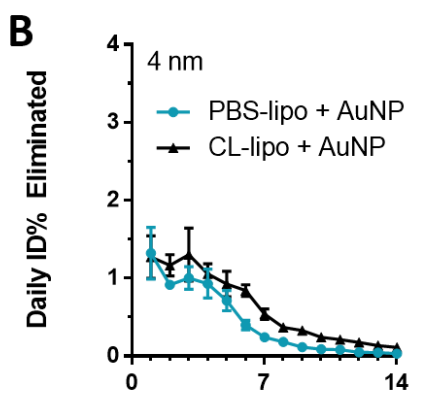

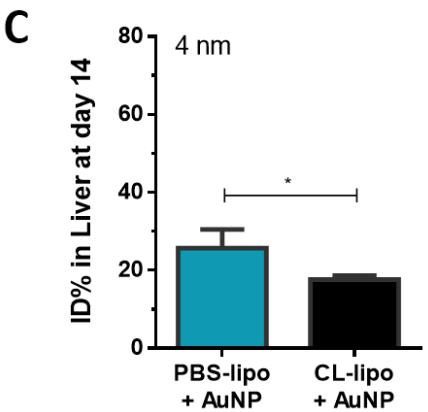

E

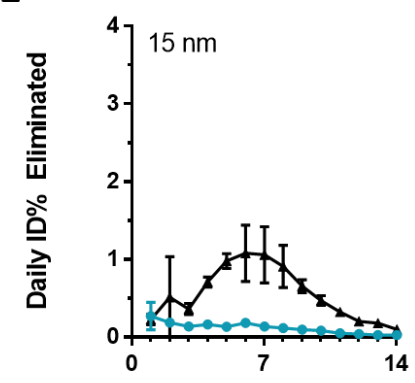

$\mathbf{F}$

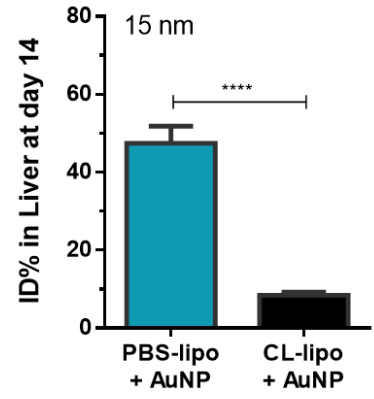

H

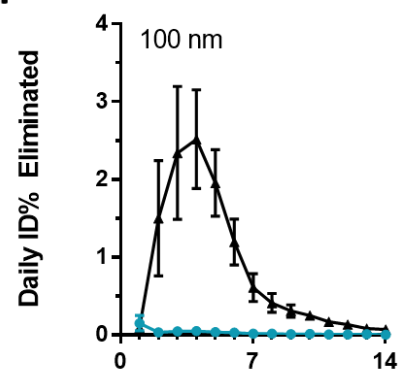

K

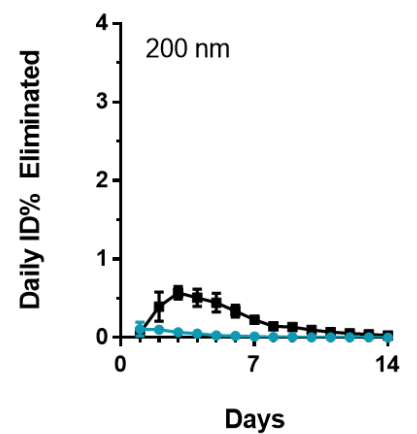

I

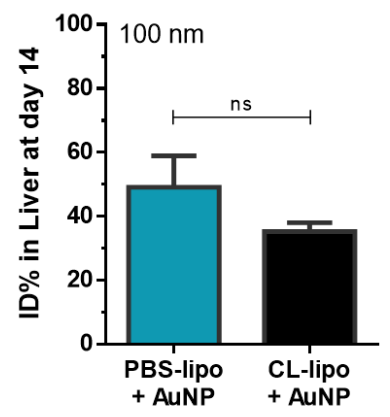

$\mathbf{L}$

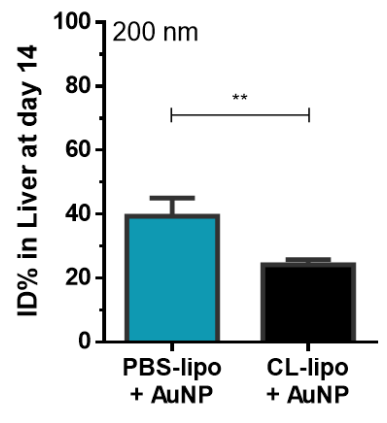

Fig. S15 - Quantification of how depletion of Kupffer cells improves fecal elimination of AuNPs $(A, D, G, J)$ Cumulative ID\% eliminated from BALB/C mice pre-treated with control PBS-liposomes (PBS-lipo) or clodronateliposomes (CL-lipo) over 14 days post-injection of $4 \mathrm{~nm}, 15 \mathrm{~nm}, 100 \mathrm{~nm}$, and $200 \mathrm{~nm}$ AuNPs; (B,E,H,K) Daily rate of ID\% eliminated from BALB/c mice over 14 days post-injection of $4 \mathrm{~nm}, 15 \mathrm{~nm}, 100 \mathrm{~nm}$, and $200 \mathrm{~nm}$ AuNPs; (C,F,I,L) ID\% of liver at the end of the experiment from BALB/c mice (14 days post-injection of $4 \mathrm{~nm}, 15 \mathrm{~nm}, 100 \mathrm{~nm}$, and $200 \mathrm{~nm}$ AuNPs). Data is displayed as mean \pm standard deviation with a sample size of $n=3-4$. Statistics determined by unpaired $t$-test with $n s=n o t$ statistically significant, ${ }^{*}=p<0.05,{ }^{* *}=p<0.01,{ }^{* * *}=p<0.001$, and ${ }^{* * *}=p<0.0001$. 
A

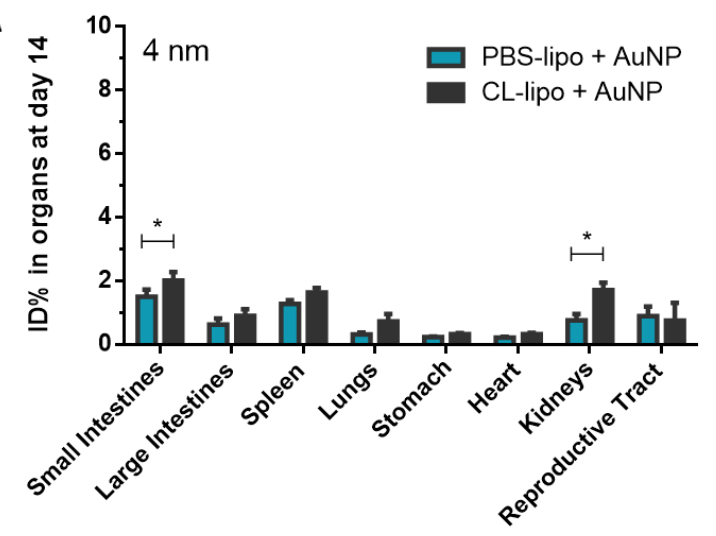

C

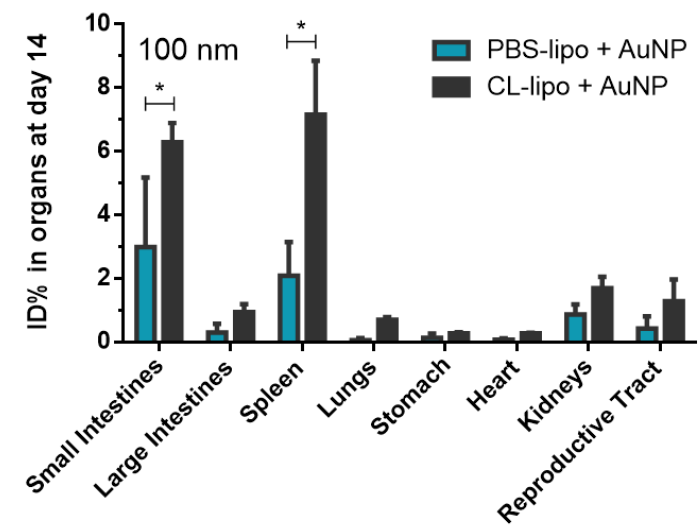

B

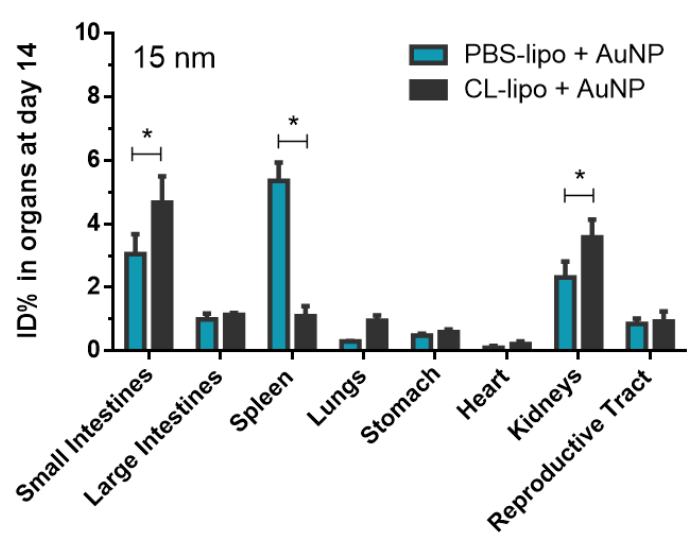

D

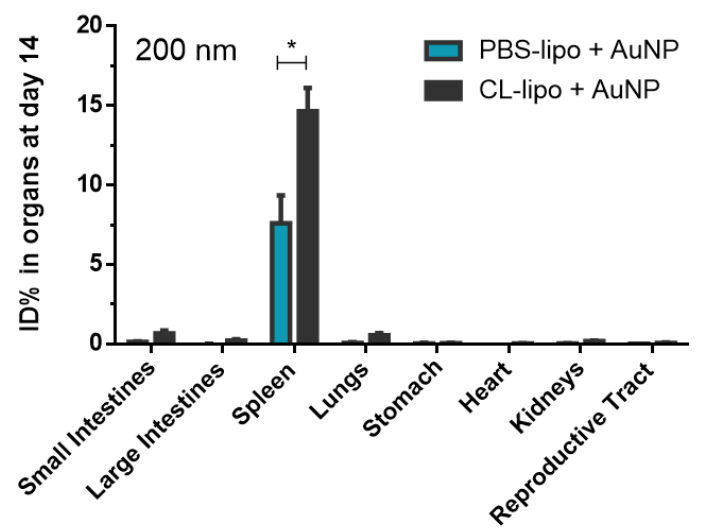

Fig. S16 - Organ-level distribution of different AuNP sizes for control and pre-depletion groups

(A) ID\% of AuNPs in each major organ of BALB/c mice 14 days post-injection of $4 \mathrm{~nm}$ AuNPs for control PBS-liposome (PBS-lipo) and clodronate-liposome (CL-lipo) pre-depletion groups; (B) ID\% of AuNPs in each major organ of BALB/C mice 14 days postinjection of $15 \mathrm{~nm}$ AuNPs for control and pre-depletion groups; (C) ID\% of AuNPs in each major organ of BALB/c mice 14 days post-injection of $100 \mathrm{~nm}$ AuNPs for control and pre-depletion groups; (D) ID\% of AuNPs in each major organ of BALB/c mice 14 days post-injection of $200 \mathrm{~nm}$ AuNPs for control and pre-depletion groups. Data is displayed as mean \pm standard deviation with a sample size of $n=3-4$. Statistics determined by multiple t-test with Sidak-Bonferroni correction, ${ }^{*}=p<0.05$. 


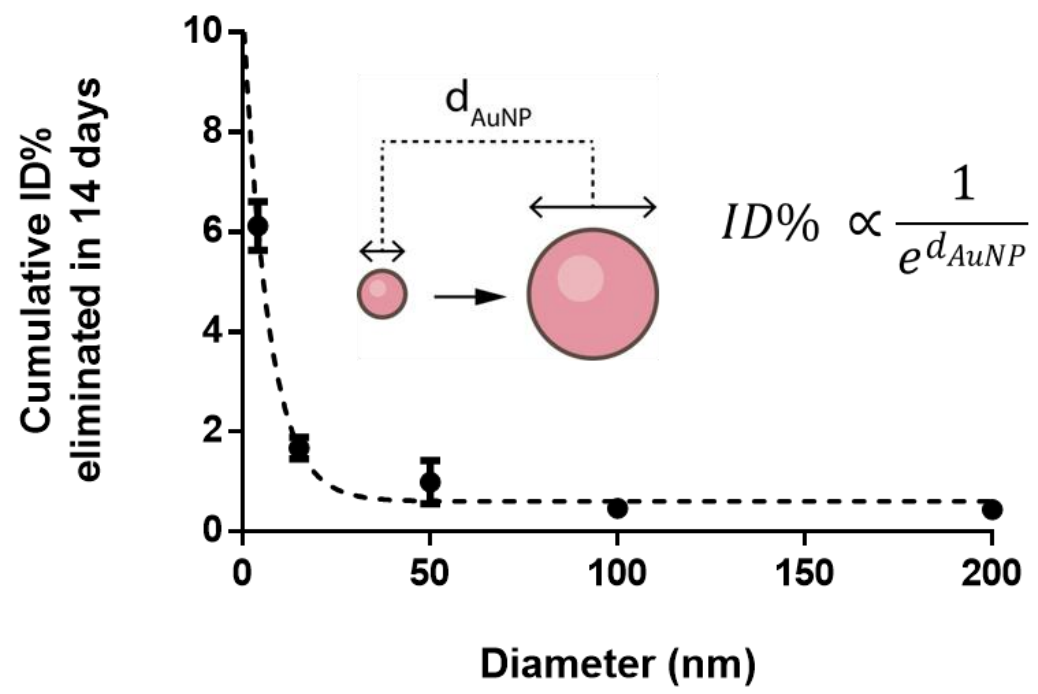

Fig. S17 - Cumulative AuNP ID\% eliminated in 14 days in control BALB/c mice is a function of AuNP size The cumulative ID\% eliminated in 14 days of AuNPs in control BALB/C mice is dependent on AuNP size, with an exponential decay mathematical relationship. Data is displayed as mean \pm standard deviation with a sample size of $n=3-4$.
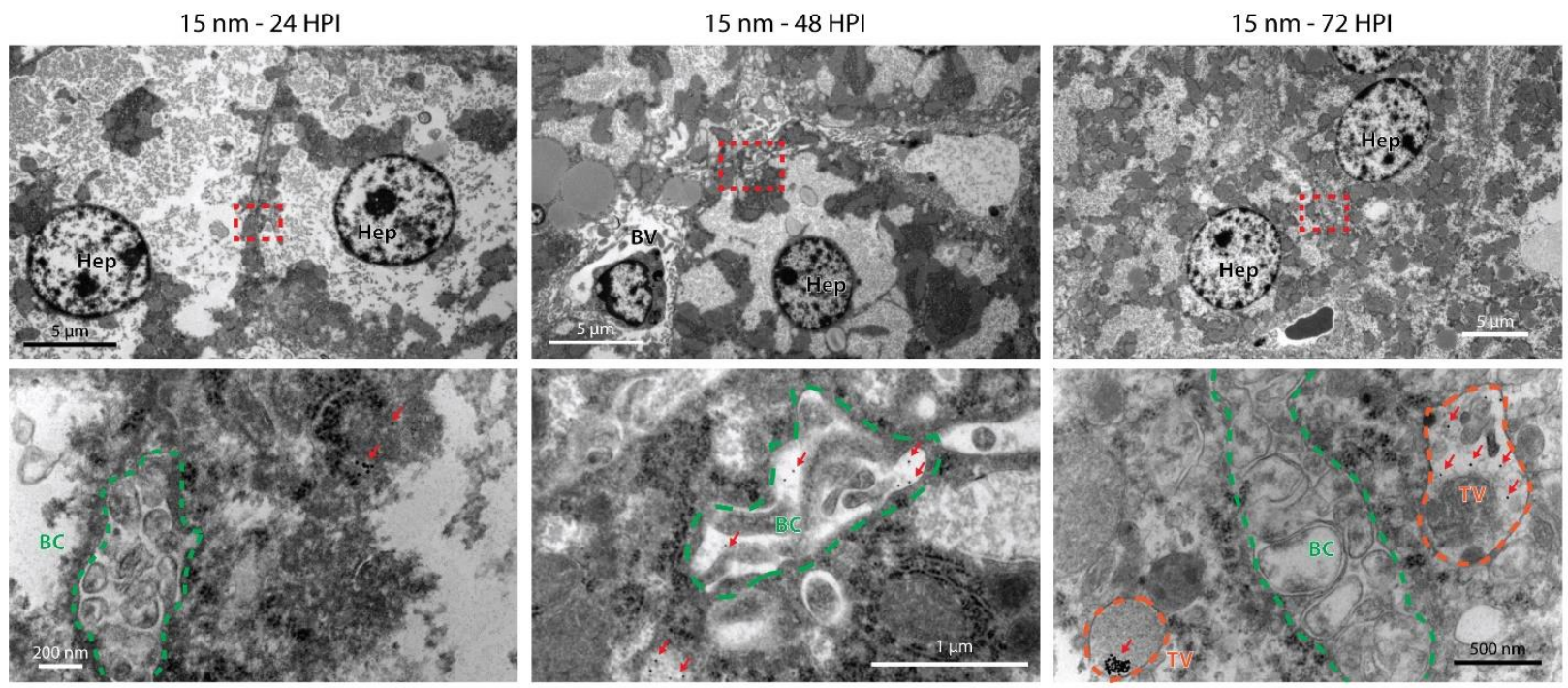

Fig S18 - Subcellular visualization of $15 \mathrm{~nm}$ AuNPs undergoing hepatobiliary processing by TEM

(Left to right) Representative TEM images of livers from BALB/c animals pre-treated with clodronate-liposomes at 24 hours, 48 hours, and 72 hours post-injection of $15 \mathrm{~nm}$ AuNPs. Bottom panels are magnified regions of the corresponding areas indicated by the red dotted squares. Red arrows indicate locations of nanoparticles. Black triangle pairs indicate the occluding belt between adjacent hepatocytes. $B V=$ blood vessel, $B C=$ bile canaliculus, $T V=$ transport vesicle, Hep = hepatocyte. 

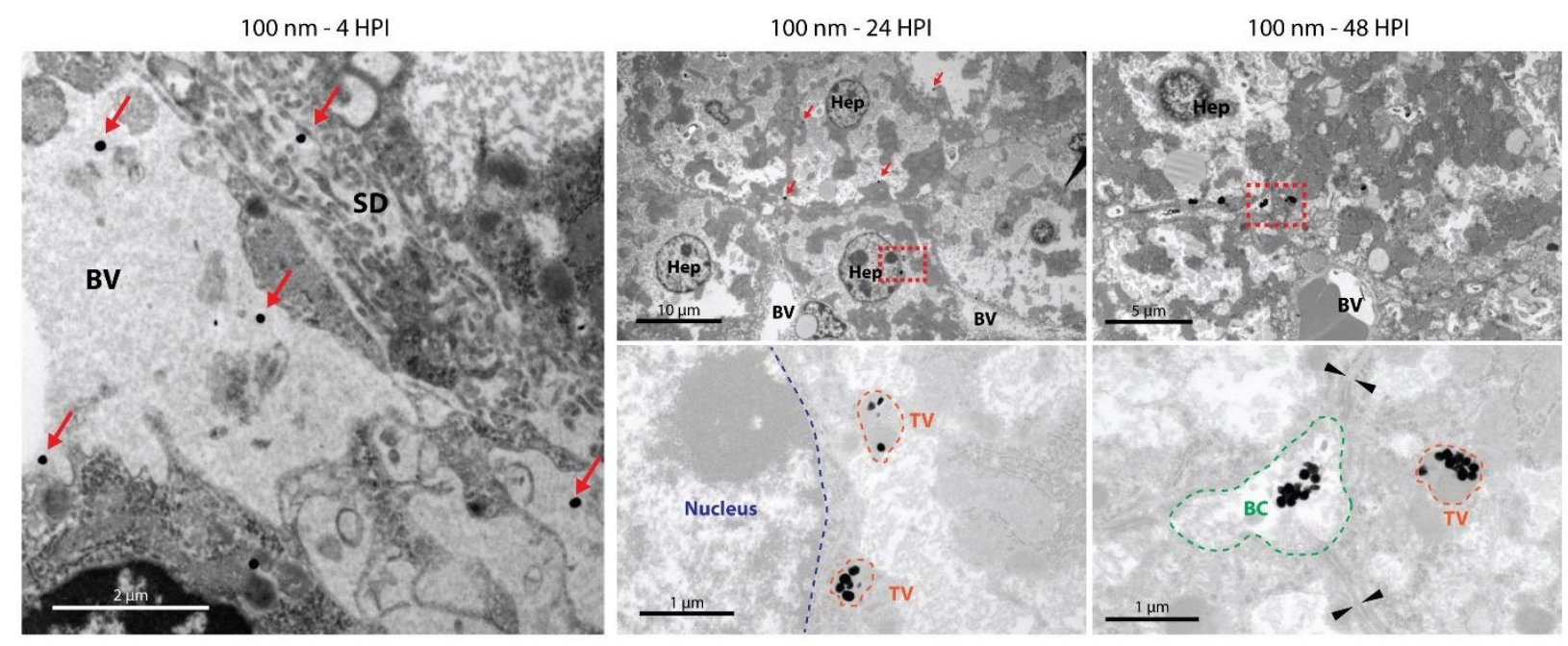

Fig S19 - Subcellular visualization of $100 \mathrm{~nm}$ AuNPs undergoing hepatobiliary processing by TEM

(Left to right) Representative TEM images of livers from BALB/C animals pre-treated with clodronate-liposomes at 4 hours, 24 hours, and 48 hours post-injection of $100 \mathrm{~nm}$ AuNPs. Bottom panels are magnified regions of the corresponding areas indicated by the red dotted squares. Red arrows indicate locations of nanoparticles. Black triangle pairs indicate the occluding belt between adjacent hepatocytes. $B V=$ blood vessel, $S D=$ space of Disse, $B C=$ bile canaliculus, $T V=$ transport vesicle, Hep $=$ hepatocyte.

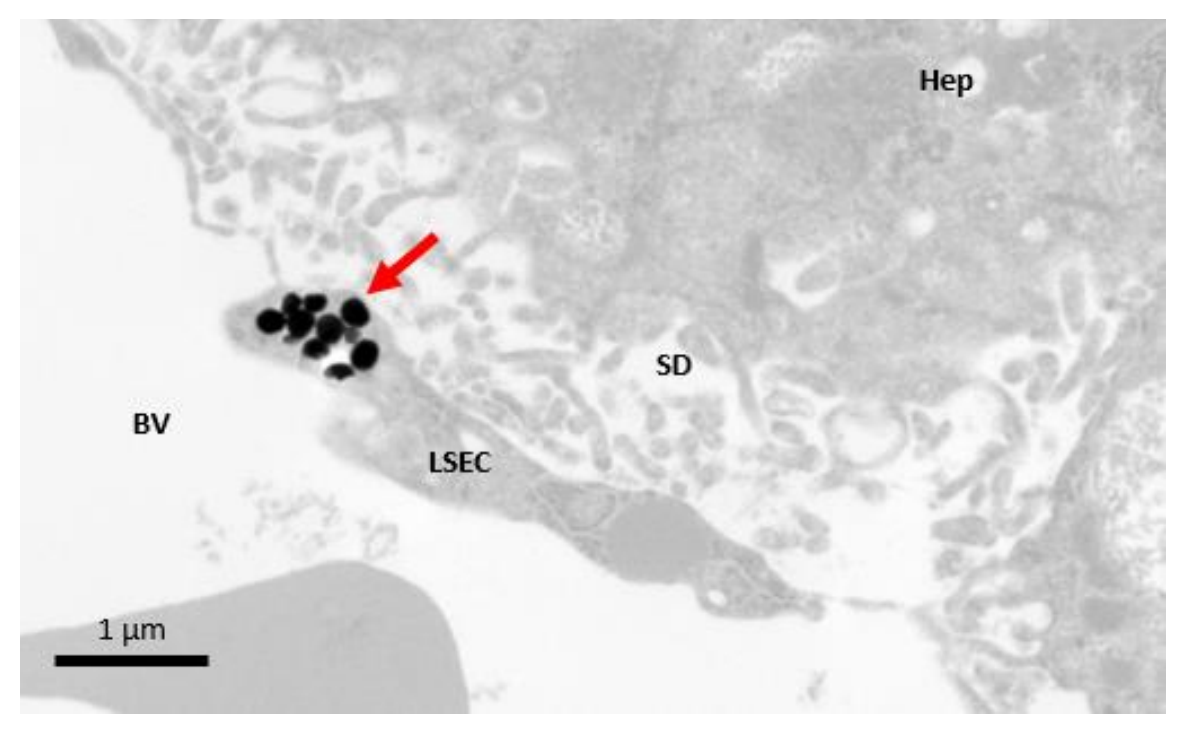

Fig S20 - Subcellular visualization of $200 \mathrm{~nm}$ AuNPs in liver sinusoid by TEM

TEM images of a liver sinusoid from BALB/c mouse 14 days post-injection of $200 \mathrm{~nm}$ AuNPs. Red arrow indicates location of nanoparticles. $B V=$ blood vessel, $S D=$ space of Disse, Hep = hepatocyte, $L S E C=$ liver sinusoidal endothelial cell. 


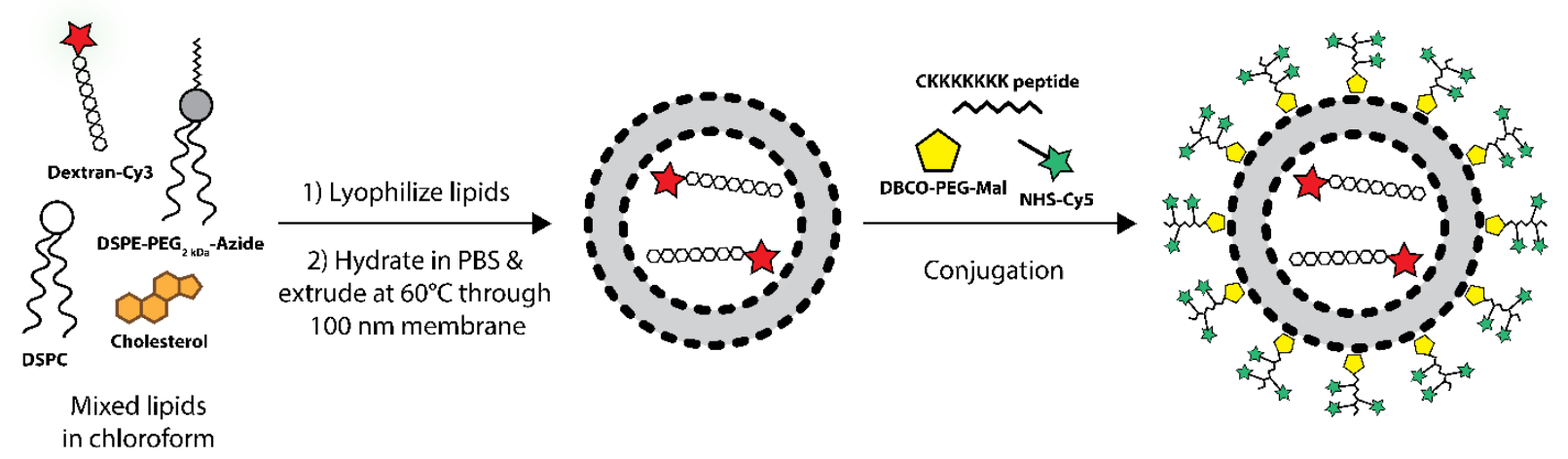

Fig S21 - Characterization and synthesis of Cy3/Cy5 dual-labelled degradable liposomes

Synthesis of Cy3/Cy5 dual-labelled degradable liposomes is shown above, characterization by DLS shows that liposomes have hydrodynamic diameter of $135 \mathrm{~nm}$ and a polydispersity index of 0.09. Abbreviations: DSPC = 1,2-distearoyl-sn-glycero-3phosphocholine; DPSE-PEG 2 kDa-Azide = 1,2-distearoyl-sn-glycero-3-phosphatidylethanolamine-N-[azido(polyethylene glycol)2000]; DBCO-PEG-Mal = dibenzocycloctyne-[(polyethylene glycol)-4]-maleimide; $N H S=N$-hydroxysuccinimide 